Clio. Femmes, Genre, Histoire

19 | 2004

Femmes et images

\title{
Le système des objets dans le testament de Blanche de Navarre
}

\section{Brigitte Buettner}

\section{(2) OpenEdition \\ 1 Journals}

Édition électronique

URL : http://journals.openedition.org/clio/644

DOI : 10.4000/clio.644

ISSN : 1777-5299

Éditeur

Belin

Édition imprimée

Date de publication : 1 avril 2004

ISSN : 1252-7017

Référence électronique

Brigitte Buettner, "Le système des objets dans le testament de Blanche de Navarre », Clio. Femmes, Genre, Histoire [En ligne], 19 | 2004, mis en ligne le 27 novembre 2006, consulté le 01 mai 2019. URL: http://journals.openedition.org/clio/644 ; DOI : 10.4000/clio.644

Ce document a été généré automatiquement le 1 mai 2019.

Tous droits réservés 


\title{
Le système des objets dans le testament de Blanche de Navarre
}

\author{
Brigitte Buettner
}

\section{NOTE DE L'ÉDITEUR}

Que François Avril, Joan Holladay, Rita Keane, Christiane Klapisch-Zuber et Catherine de Smet soient vivement remerciés pour l'aide qu'ils ont bien voulu apporter.

Blanche de Navarre, surnommée Belle Sagesse, devint reine de France en 1350 lorsqu'elle épousa Philippe VI de Valois; celui-ci mourut à peine quelques mois plus tard, la laissant enceinte d'une fille. Blanche, quant à elle, allait vivre encore un demi-siècle, quittant ce monde en 1398 à l'âge avancé de 67 ans - veuve riche et respectée, ayant derrière elle, aux dires de la Chronique de Charles VI, une vie passée dans la chasteté, protégeant veuves, orphelines et pauvres, à tel point que son hôtel tenait plus d'un « cloître de religieux que d'un palais de reine $»^{1}$. Mis à part sa longévité et la défense, parfois tenace, de ses biens et privilèges, qui lui incombait comme à toute veuve au Moyen Âge, mis à part aussi le rôle politique qu'elle joua (et sur lequel nous aurons à revenir), la biographie de Blanche - ou mieux, les quelques lambeaux qui nous en sont parvenus - ne présente rien de vraiment exceptionnel. En particulier, et bien qu'elle ait commandité quelques œuvres, surtout à caractère pieux ${ }^{2}$, son nom ne se laisse rattacher à aucun de ces nombreux manuscrits enluminés créés pour des femmes de la haute noblesse française tout au long du XIVe siècle, comme, et pour ne mentionner que ceux-là, les célèbres Heures enluminées par Jean Pucelle pour sa tante Jeanne d'Évreux ou le tout aussi magnifique livre d'heures exécuté pour sa mère Jeanne II de Navarre ${ }^{3}$. Alors pourquoi la considérer dans le contexte de la relation des femmes aux images?

2 C'est que Blanche a tout de même une originalité, celle d'avoir rédigé un testament remarquable par son détail et son élaboration, et dont nous avons la chance qu'il nous soit parvenu. Tant la quantité que la qualité des objets lui ayant appartenu sont inhabituelles et n'ont pas d'égales dans les collections des hommes de son temps. Mais 
l'intérêt du document, publié en 1885 par Léopold Delisle, le grand savant spécialiste des manuscrits médiévaux, réside non seulement dans ce qu'il révèle des biens possédés par une femme privilégiée de la fin du Moyen Âge, mais aussi dans ce qu'il dit sur la façon dont elle avait rassemblé ses avoirs les plus précieux et décida de s'en dessaisir à la veille de son trépas. Ce dernier aspect surtout retiendra notre attention, car il invite, sinon à revoir la manière dont nous entendons aujourd'hui le rapport des femmes aux images, du moins à en déplacer quelque peu les termes en nous interrogeant sur ce que constituait au juste le visuel pour Blanche et sur ce que furent les termes de son mécénat. Il s'agira surtout de voir si la distinction que nous faisons entre manuscrits enluminés et objets d'art (distinction qui correspond à des compétences professionnelles différentes) pouvait être retenue par Blanche ou si, au contraire, ces œuvres n'étaient pas alignées sur un même axe esthétique où les lignes de partage pertinentes n'étaient pas celles entre image et objet mais suivaient d'autres critères. Par ailleurs, on pourra se demander si notre approche habituelle du mécénat, entendu comme l'étude d'une commande passée entre deux individus vivants, n'est pas inadéquate pour rendre compte du rapport que beaucoup de femmes, et pas seulement au Moyen Âge, entretenaient avec des biens culturels. Sans sous-estimer le rôle du mécénat proprement dit, j'aimerais dire d'emblée que si l'on s'attache à lui seulement, on risque de jeter un regard trop moderne, voire même trop masculin sur des femmes qui firent un art de la circulation de choses, et pas seulement de leur création.

\section{Reine douairière}

3 Fille de Philippe III d'Évreux et de Jeanne II de Navarre, Blanche descendait du côté maternel de la branche aînée issue de Louis IX, Philippe le Bel étant son arrière-grandpère ${ }^{4}$. Sa mère avait du reste été celle qui s'était vue écartée de la succession à la couronne lors de la mort de son père, Louis X le Hutin, et avait été remplacée, en vertu de la règle qui plus tard devait prendre le nom de loi salique, par son oncle, Philippe $\mathrm{V}$ le Long. Par ailleurs, Jeanne avait aussi été contrainte de céder la Champagne et la Brie en échange de la reconnaissance de son droit à régner sur la Navarre et de quelques seigneuries en Normandie, territoires qu'il était beaucoup moins gênant pour le domaine royal de perdre. Ces deux décisions furent la source nourrissant l'opposition acharnée du frère de Blanche, Charles II de Navarre, à Jean le Bon et à Charles V, opposition souvent armée, qui plusieurs fois mena la France au bord de la guerre civile. Rappelons que Charles est passé à l'histoire, depuis le XVIe siècle, avec le surnom de Mauvais, tout en ajoutant que ce n'est pas sans justice qu'il estimait que le trône de France aurait dû lui échoir plutôt qu'à ses cousins de la branche cadette des Valois 5 . À l'occasion de l'un des innombrables et tout aussi provisoires rapprochements avec les Valois, Charles négocia avec le premier roi de la nouvelle dynastie, Philippe VI, le mariage de Blanche, les deux hommes décidant qu'elle serait donnée au fils aîné du roi, Jean, duc de Normandie, futur Jean II le Bon, dont la première femme, Bonne de Luxembourg, venait de succomber aux ravages de la peste. Puis, et malgré quelques différences dans le détail, les chroniqueurs s'accordent sur le fait que dès son arrivée à Paris en 1349, année où la peste avait aussi fauché la vie de la reine Jeanne de Bourgogne, ce fut en fait le vieux roi empâté, de quarante ans son aîné, de surcroît son oncle au troisième degré, qui décida de faire de Blanche son épouse, sans doute ébloui par sa beauté mais certainement tout aussi séduit par l'idée de s'unir à l'une de celles qui étaient plus capétiennes que lui-même, savourant 
peut-être jusqu'à l'ironie qu'il devait le fait d'être roi de France à l'exhérédation de la mère de la jeune fille.

Blanche passa les longues années de son veuvage soit à Paris, dans sa maison rue de la Tissanderie ou dans l'un des couvents qu'elle affectionnait, soit dans les demeures de son douaire, celles de Melun d'abord, de Neauphle-le-Château ensuite. C'est là, avant le vide de ses dernières années, que se trouvait rassemblé un véritable gynécée comprenant, outre sa fille Jeanne, sa sœur puînée, Jeanne dite la Jeune (qui part en 1373 épouser le Vicomte de Rohan), sa belle-sœur Jeanne (sœur de Jean II le Bon et femme de Charles de Navarre) et surtout une autre Jeanne encore, sa tante Évreux, elle aussi reine douairière et veuve pendant la plus grande partie de sa vie, et elle aussi femme d'un roi de France. Blanche était une sorte de seconde fille pour elle, et c'était comme à une sœur qu'elle était liée à son seul enfant, une Blanche aussi, tout à la fois cousine et belle-fille puisqu'elle avait épousé le très insignifiant Philippe d'Orléans ${ }^{6}$. Même à l'écart du centre de gravité de la cour de France, c'est un petit clan puissant, uni par de multiples liens de parenté très proches, par la conscience aussi de se rattacher plus directement que les Valois à l'ancêtre mythique, saint Louis, le « patron de la dynastie » par rapport auquel se définissaient toutes les hiérarchies, tout le paysage mental de la noblesse dans la France du XIVe siècle ${ }^{7}$. Bien sûr, ces femmes se savaient en même temps étroitement liées aux Valois, mais, si besoin était, elles pouvaient leur tenir tête en se repliant sur leur propre lignage, quitte à se voir accuser d'embrasser le " parti navarrais $»^{8}$.

5 Finalement, on a le sentiment d'avoir affaire à une sorte de laboratoire social au féminin où s'aiguisaient les outils nécessaires à la gestion et à la défense du patrimoine, du douaire surtout ${ }^{9}$; où s'échafaudaient des modèles sociaux ajustés à des femmes au statut social influent; où se partageaient et se transmettaient des pratiques religieuses, culturelles et artistiques. Y compris face à la mort, car dans son testament Blanche exige à plusieurs reprises que l'on suive les dispositions adoptées, vingt-cinq ans auparavant, pour les obsèques de Jeanne d'Évreux, ultime hommage à celle qui avait été un point de référence majeur sa vie durant. Jeanne et Blanche se rejoignent enfin dans le fait véritablement exceptionnel d'avoir érigé des chapelles funéraires - plutôt que de simples tombes - dans la nécropole officielle des rois (et beaucoup moins, des reines) de France, à Saint-Denis. Espaces où elles reposent avec leurs filles respectives, et dans lesquels d'ambitieux monuments funéraires incluant des gisants en marbre et de nombreuses sculptures généalogiques affichent leur appartenance à la maison de Navarre-Évreux, et où elles revendiquent encore l'ascendance de saint Louis. Que Blanche de Navarre ait été contrainte de ré-imaginer le lieu de sa sépulture - car, comme elle le dit dans son testament, « nostre corps ne peut estre bonnement miz ne enterré de lez nostre très chier seigneur et espoux le roy Phelipe, que Dieu absoille, où il n'a pas de place pour ce faire » (510) ${ }^{10}$ - ne diminue en rien la hardiesse de son geste. Au demeurant, celui-ci est revendiqué dans la très belle charte par laquelle, en 1372, elle fonde deux messes quotidiennes en perpétuité pour les âmes de son mari, de sa mère et de son père, de sa fille et d'elle-même dans la chapelle Saint-Hippolyte - saint du jour auquel était mort Philippe de Valois. Le document s'ouvre par une délicate initiale B tracée à l'encre (Fig. 9). Blanche y est présente deux fois, d'abord par le biais de ses armoiries adossées à la lettrine, ensuite à l'intérieur, agenouillée devant saint Denis et saints Pierre et Paul, auxquels elle présente un modèle de sa chapelle, entourée de ceux qui n'étaient déjà plus : sa fille Jeanne, morte un an auparavant, et, sur la droite, un peu à l'écart, Philippe, gardé par saints Jean Baptiste et Hippolyte ${ }^{11}$. 


\section{Tisser des liens}

$6 \quad$ Notre petite tribu féminine se regroupe enfin autour d'une stratégie politique commune : assurer le rôle d'intermédiaire entre Valois et Navarre. Tous les observateurs contemporains soulignent leurs efforts répétés, que ce soit personnellement, par courrier ou par ambassadeurs interposés, pour négocier entre intérêts et individus opposés, leur engagement à amener la paix entre fratries déchirées. Certes, cette fonction d'intermédiaire, que les femmes en tant que sœurs et épouses sont amenées à occuper structurellement, avait depuis longtemps correspondu à la fois à leur rôle réel et à leur image idéale. À nos yeux, elle est l'indice d'un manque, mesurée qu'elle est à l'aune du pouvoir « réel » qui, lui, est toujours pensé au masculin. C'est oublier que la diplomatie offrait l'un des champs d'action où les femmes, au croisement du privé et du public, pouvaient échapper aux limitations imposées à leur état, où d'intermédiaires passives elles pouvaient s'ériger en actives médiatrices. Charles $\mathrm{V}$ le dit bien dans une lettre confirmant l'un des maints traités de paix avec Charles de Navarre, paix que les deux reines « eussent et aient requiz et fait requerir pluseurs foiz a grant instance [...] et tant y aient travaillé noz dites dames que, par le moien et a la priere d'icelles fu [...] fait [...] certain traitie $[. . .]^{12}$ ». Et c'est précisément la manière dont les Grandes Chroniques de France , dans la copie somptueusement enluminée pour Charles V, les met en scène (Fig. 10). Introduit par le tandem Jeanne d'Évreux et Blanche de Navarre, on y voit le repentant Charles de Navarre recevoir le pardon de Jean le Bon pour avoir commandité le meurtre du connétable Charles d'Espagne en 1354, réconciliation solennellement formalisée un an plus tard au Louvre et dont les deux reines avaient été parmi les principaux artisans ${ }^{13}$. Même si Blanche et Jeanne sont visuellement à peu près indifférenciables, leur insertion dans cette historiographie officielle qui fait généralement peu de cas des femmes si ce n'est en tant qu'épouses ou filles, confirme leur importance, tant objective que dans la perception qu'en avait Charles V.

7 Il n'est pas surprenant que Blanche, comme elle l'avait fait tout au long de sa vie, ait saisi l'occasion de la rédaction de son testament (1396) et des deux codicilles (1396 et 1398) pour mettre en œuvre une politique de réconciliation comparable - sauf que ce sont des objets qui maintenant devront forger des liens entre générations, lignages et parents, entre vivants et morts. Unique dans sa spécificité - en cela Blanche fait mieux que sa tante - le testament se conforme sinon aux articulations typiques de ce genre d'actes à la fin du Moyen Âge ${ }^{14}$. Ainsi, la testatrice "saine de corps, d'entendement et de pensée » recommande son âme à la Trinité et à la Vierge avant d'en venir aux détails concernant ses funérailles - simples, sans pompe spéciale, elle sera, si possible, transportée «à visage découvert ». Pareille restriction pour le luminaire, ce qui amène l'auteur de la Chronique $d u$ règne de Charles VI à en dénoncer le côté mesquin, à peine digne d'une personne de condition humble, s'empressant d'en accuser (injustement) les exécuteurs testamentaires; car comment expliquer autrement cette modestie, vu les «immenses trésors laissés par la reine» ${ }^{15}$ ? L'aspect le plus inattendu concerne la manière dont Blanche décide de disposer de sa dépouille, car elle insiste très fermement sur sa volonté que son corps soit enterré tout entier dans la chapelle Saint-Hippolyte, à côté de sa fille, "sanz faire nostre corps aucunement diviser, ne en icellui faire aucune incision", récusant ainsi la pratique princière qui continuait à favoriser le partage du corps, avec 
entrailles, cœur et os séparés de la chair et disséminés en trois lieux de sépulture différents ${ }^{16}$.

8 En sus des sommes d'argent considérables que Blanche réserve pour ses funérailles, les messes commémoratives, les dons charitables et legs pieux, elle témoigne sa reconnaissance envers les églises et couvents qui lui tenaient le plus à cœur en leur remettant un objet précieux, reliquaire ou ymage (statuette). Bien qu'aucun ne nous soit parvenu, les descriptions soigneuses laissent entendre qu'il devait s'agir d'œuvres tout aussi raffinées que le merveilleux reliquaire de la Vierge à l'Enfant, en argent doré, offert par Jeanne d'Évreux à l'abbaye de Saint-Denis (aujourd'hui au Louvre). Si l'on ajoute à ces objets ceux qu'elle réserve à sa parenté (de sang, d'alliance et, dans quelques cas, spirituelle) ainsi qu'aux plus importantes personnes à son service, et sans tenir compte des choses de nature plus humble ${ }^{17}$, on obtient le volume considérable de vingt-deux reliquaires et ymages sacrées; cinq «chapelles " (vêtements et ornements liturgiques); quatre tableaux sur bois ; quarante manuscrits ; vingt-deux joyaux ; et onze " chambres » (garnitures de lit). Notons que parmi ces objets de prestige le nombre de reliquaires et de livres est particulièrement élevé. Pour ces derniers, on peut comparer la bibliothèque de Blanche à celle de la reine Clémence de Hongrie, inventoriée après son décès en 1328, et qui comptait environ quarante livres, ou à celle de Jeanne d'Évreux qui, selon les estimations récentes de Joan Holladay, s'élevait à quarante-neuf volumes au minimum ${ }^{18}$. Ces collections féminines étaient bien plus étendues que celles des époux, et leur contenu se ressemblait aussi, avec une prépondérance d'ouvrages à caractère religieux adaptés à l'usage privé - tels les bréviaires (livres comprenant les prières à réciter aux huit heures canoniques), les psautiers (contenant les 150 psaumes), et les livres d'heures (le livre de prière le plus répandu à la fin du Moyen Âge) - et des traités de dévotion ou de la littérature didactique. Les œuvres profanes y sont plus rares mais ne manquent jamais. Dans le cas de Blanche, on trouve, par exemple, le Livre du gouvernement des princes de Gilles de Rome, les Grandes Chroniques de France, les Chroniques d'Outremer (une histoire de la Terre Sainte écrite par Guillaume de Tyr avant 1184), le Déduit des chiens et des oiseaux (un traité sur la chasse à fond moral composé sur ordre de Jean le Bon par de Gace de la Buigne, jadis chapelain de Philippe VI), ou encore l'encyclopédie en prose française très en vogue connue comme le Livre de Sydrac.

\section{La généalogie des objets}

9 C'est bien parmi les vingt-trois individus de sa parenté que Blanche a particulièrement investi son énergie, distribuant avec un entrain méticuleux ses biens les plus importants, «jouant à Dieu» en quelque sorte, comme Martha Howell l'a bien dit à propos des femmes de Douai dont elle a analysé les testaments ${ }^{19}$. Blanche se conforme à la règle habituelle qui veut que les premiers dons aillent au roi, Charles VI, et à la reine, Isabeau de Bavière. Elle réserve deux objets à chacun, comme elle le fait pour la plupart de ses héritiers, à commencer par les autres membres de la famille royale restreinte - les oncles du roi, Jean de Berry et Philippe le Hardi et son frère, Louis d'Orléans. Par contraste, Marie, reine de Sicile, épouse et en quelque sorte remplaçante de Louis Ier d'Anjou, troisième oncle, défunt, de Charles VI, ne reçoit qu'un livre, sans doute important puisque Blanche le décrit comme «nostre breviare le milleur» (204). Un seul objet également - un chapel, c'est-à-dire une couronne ou un cercle d'or semé de pierres (20520) - pour Isabelle de France, qui était à la fois arrière-arrière-petite-fille et filleule de Blanche. Au sein du 
premier groupe composé d'affins proches, seul se détache son neveu Charles III, l'actuel roi de Navarre. Non seulement en vertu des règles de préséance il est mentionné immédiatement après le roi et la reine de France, mais il lui échoit quatre objets, y compris l'un des deux livres les plus importants que Blanche possède, le psautier de saint Louis, sur lequel je reviendrai. Suivent les parents par alliance plus éloignés : Louis II, duc de Bourbon, doyen à côté de Blanche de la cour de France; les épouses des oncles paternels du roi ainsi que Marie, duchesse de Bar, tante paternelle de Charles VI (alors que les tantes maternelles ne sont pas incluses). Et pour finir, le groupe plus varié du propre lignage de Blanche, les Évreux-Navarre, cinq femmes et trois hommes: les cousins, les sœurs et quelques nièces et neveux, suivis d'une cousine plus éloignée, peutêtre même nominale, la dame de Préaux ${ }^{20}$.

10 Les dons s'étendent donc sur quatre générations, Isabelle de France étant la plus éloignée au moment de la rédaction du premier codicille, mais recevant, lors du second, la compagnie de Blanche, née en 1391, fille de Charles III de Navarre (nommée en l'honneur de sa grande-tante, on la connaît maintenant comme Blanche II de Navarre). En revanche, l'extension latérale est réduite, ce qui montre que Blanche concentre ses legs sur les branches principales des Valois comme des Navarre. Mais ces parents, pourtant assez proches, ne se valent pas, et l'on voit Blanche manier plusieurs variables pour introduire gradations et différences dans l'équivalence. Ainsi utilise-t-elle d'abord la place de l'individu dans l'énumération (exception faite de Charles de Navarre, les affins d'abord, les consanguins ensuite); puis la désignation des individus, puisque trois seulement, Charles VI, Charles de Navarre et Louis de Bourbon sont qualifiés de «tres amé » en sus de l'habituel «tres chier »; le nombre des objets, enfin (en moyenne deux pour ses affins, de trois à cinq pour ses consanguins); et si elle considère moins le type de ces objets, étant donné que presque tous ses héritiers reçoivent au minimum un livre et un joyau, elle tient compte aussi de leur qualité, encore qu'il soit difficile d'en donner une estimation même approximative en raison de l'absence d'évaluations monétaires.

11 Reste un dernier critère, en fait le plus significatif pour Blanche, à savoir la provenance des objets que son testament mentionne souvent et, chose rare, de manière parfois très détaillée ${ }^{21}$. Hormis quelques articles d'origine disparate, ses objets proviennent de deux sources principales. La première est Jeanne d'Évreux, directement ou indirectement - par le truchement de leurs filles notamment -, ce qui explique que plusieurs objets fassent divers va-et-vient, comme le très beau patenôtre donné à la duchesse de Bourgogne (210) ou l'anneau garni d'un rubis légué à la duchesse de Berry $(208)^{22}$. La seconde source est son mari, bien que dans plusieurs cas celui-ci ait hérité les objets de sa première femme avant de les offrir à Blanche. Redéployer des biens entre femmes successives était pratique courante, indice d'une certaine interchangeabilité entre épouses, manière aussi d'assurer la continuité d'un statut par-delà les individus particuliers, témoignage, enfin, d'un rapport différent aux choses, qui semblent avoir acquis un surplus de valeur à mesure qu'elles circulaient. Cependant on peut se demander si l'empressement avec lequel est mentionné « madame la royne Jehanne de Bourgongne (sic) » ne trahit pas une motivation supplémentaire: il s'agit peut-être pour Blanche, face à sa propre mort, d'expier la culpabilité d'avoir pris la place de la défunte auprès de son mari et de ses nombreux enfants.

12 Toutefois, la filiation d'un objet se fait souvent plus précise, plus tortueuse aussi, quand la mémoire remonte jusqu'au propriétaire originel (ou jugé tel). Blanche utilise alors ces lignes de transmission complexes pour mettre l'accent sur les relations lignagères plutôt 
que simplement établir un trait d'union entre générations. Ainsi, il lui importe que les membres de la famille royale deviennent les dépositaires des objets qui avaient appartenu à son mari et à sa première femme, c'est-à-dire à leurs ancêtres directs et au chef de lignage, comme si elle voulait restituer ces reliques personnelles qui ne lui avaient été prêtées à elle-même que sa vie durant ${ }^{23}$. Charles VI, par exemple, se voit assigner une ceinture « de bisete [galon étroit] d'or trait à losenges de perles aux armes de France à lys d'or, qui se euvrent, et y a pluseurs bonnes reliques ", ceinture dont Jeanne de Bourgogne avait fait cadeau à son mari et que celui-ci, Blanche le note, portait comme un talisman lorsqu'il partait à la guerre (192); et aussi un «signet » (= sceau) qui, par-delà Philippe, remonte à Charles IV (le Bel), simplement identifié comme " père de nostre très chère fille la duchesse d'Orléans » (193). Notons que c'est un menu objet, lourd de sens, qui se voit chargé de relier le présent roi Valois au dernier Capétien direct, façon d'effacer la rupture dynastique, à moins qu'il ne s'agisse, au contraire, de la rappeler à celui qui allait désormais porter ce sceau à son propre doigt. L'un des deux objets légués à Jean de Berry, un petit diamant (199), descend pareillement de Philippe VI, qui dans ce cas l'avait reçu de sa sœur, Jeanne de Valois, comtesse de Hainaut, elle aussi une infatigable médiatrice, notamment entre son frère et son beau-fils, Edouard III, l'autre principal prétendant au trône de France. Quant au duc de Bourgogne Philippe II le Hardi, l'homme fort du moment, il se voit octroyer, en sus d'un fermail d'or que son grand-père portait «à sa poitrine » et auquel il attachait des reliques ${ }^{24}$ (voir Fig. 11), le deuxième manuscrit majeur que Blanche possède, muni lui aussi d'une longue mémoire lignagère puisque ce «psaltier où monseigneur saint Loys aprint» remonte à six générations ${ }^{25}$. La très fine intelligence que Blanche a des rapports entre personnes et choses se révèle particulièrement bien dans cet exemple, en ce sens que ce livre précieux passa par la ligne féminine de Bourgogne, d'Agnès de Bourgogne à sa fille Jeanne de Bourgogne, avant d'en venir à rattacher les Valois-Bourgogne aux Capétiens-Bourgogne. En même temps, on ne peut exclure une dose d'ironie de la part de "Belle Sagesse " étant donné qu'Agnès de Bourgogne avait été la championne la plus ardente du droit de Jeanne de Navarre à succéder à la couronne de France, et que l'assignation du duché de Bourgogne que Charles $\mathrm{V}$ avait faite à son frère cadet fut contestée, encore inutilement, par Charles II de Navarre.

13 L'objet le plus significatif que Blanche transmet à son propre lignage est un livre qui fait pendant à ce psautier de saint Louis. Dans ce cas-ci, il s'agit d'un bréviaire qui «fu monseigneur le roy saint Loys de France ", livre très spécial que « l'ange lui apporta en la chartre (= prison) quant il fu pris des ennemis de la foy» (196), scène notamment représentée dans les Heures de Jeanne d'Évreux (Fig. 12). Elle laisse ce livre d'une valeur dynastique incalculable au chef actuel de la maison Évreux-Navarre, son neveu Charles III, et en note de nouveau la transmission exacte: Louis IX donna le livre à son fils Philippe III le Hardi, qui en fit cadeau à sa deuxième femme, Marie de Brabant, «nostre besaiole ", et le bréviaire passa ainsi "de hoir en hoir de la ligniée monseigneur saint Loys » jusqu'à Charles II de Navarre, qui s'en était défait en faveur de sa sœur, laquelle veut maintenant le restituer à la ligne mâle aînée. Le parallèle frappant que Blanche établit entre ces manuscrits n'en fait que mieux ressortir deux différences de taille, à savoir que, là où l'exemplaire Navarre peut se vanter sinon d'une origine au moins d'une restitution miraculeuse et d'une filiation par le fils aîné de Louis IX, celui de Bourgogne doit se contenter d'être de facture humaine et de provenir d'une fille.

14 Pour celle qui était en quelque sorte la mémoire incarnée de la cour de France, celle qui s'improvisa historienne quand Charles VI l'interrogea sur le protocole à suivre lors du 
sacre et de l'entrée d'Isabeau de Bavière à Paris en $1386^{26}$, la généalogie des objets était, on le voit, leur qualité la plus marquante. Embrassant un total de neuf générations, soit à peu près deux siècles, de Blanche de Castille à Isabelle de France, sa mémoire se cristallisait autour de quelques ancêtres totémiques - saint Louis, au premier chef ${ }^{27}$ - plus quelques ascendants du côté paternel, mais aussi la mère, le mari et sa première femme, alors que le père, Philippe III d'Évreux, n'est évoqué que dans le testament proprement dit, là où Blanche laisse à l'église de Pampelune, « où gist le corps de nostre très chier seigneur et père", une "chapelle" de velours vermeil (39). Blanche peut être très explicite, voire loquace, sur la capacité des objets à s'imprégner de mémoire, et par conséquent à fournir une preuve tangible de cet intangible que sont les relations de parentéé ${ }^{8}$. D'où l'attention aux armoiries, à celles de France, de Bourgogne, et aux siennes dont, par exemple, est semée une «chambre» vermeille qu'elle substitue en 1398 à celle sans armoiries qu'elle avait d'abord réservée à sa sœur la Vicomtesse de Rohan (227 et 409). Et l'on imagine aisément l'importance que devait revêtir à ses yeux un gobelet d'or « couvert à ys gregoiz, armoiez des armes de nostre dicte dame et tante et des nostres » (241).

15 Mais c'est bien encore dans les notices se rapportant aux deux manuscrits venant de saint Louis que le motif héréditaire devient le plus net. Après en avoir retracé la transmission, Blanche fait ajouter au sujet du bréviaire qu'elle laisse à Charles III de Navarre : «Et pour reverence et la sainteté de monseigneur saint Loys, et que par grace il est venu de la ligne de nous, et depuis que nous eusmes le dit breviaire promeismes à nostre dit frère que il retourneroit en nostre ligne, nous voulons et ordonnons que à nostre dit neveu il demeure, et desormais ensuivament à ses successeurs, senz estre aucunement estrange, et les requerons que ilz le facent tousjours garder comme precieux et noble jouel venu de noz anccesseurs, et qu'il ne parte point de la lignie» (196). Tant l'histoire passée que le destin futur de cet objet mémorial sont ainsi tracés, et l'on devine la fière conscience que Blanche a d'être l'une des mailles de cette longue chaîne d'individus unis par le sang, le nom, les terres et, maintenant, par des objets, la conscience aussi d'être le pivot entre ceux qui ne sont plus et ceux qui ne sont pas encore. La teneur de l'article décrivant le psautier de saint Louis est comparable, car après en avoir évoqué le parcours qui l'amène à elle-même, Blanche précise : « si desirons qu'il soit à la ligne. Et pour ce prions à nostre dit filz [Philippe le Hardi] que il le veuille garder et faire tenir à ses successeurs et en sa ligne, pour l'amour de ceulx dont il est venu » (200). Le motif de «l'amour " pour ses prédécesseurs ou pour la testatrice revient du reste à plusieurs reprises, comme par exemple au sujet du diamant qui avait appartenu à Louis d'Évreux, «nostre ayeul », et que Blanche laisse à son neveu Pierre de Navarre, comte de Mortain, le « priant » que « le dit dyamant il vueille garder toute sa vie pour l'amour de nostre dit seigneur et de nous » (22630) et, un peu différemment, au sujet d'un reliquaire avec un clou de la Crucifixion, légué par Blanche d'Orléans qui l'avait reçu de sa mère, et que Blanche, suivant le vouloir de sa cousine, donne aux Carmes de Paris afin qu'il le gardent « comme digne saintuaire et pour l'amour d'elles et de nous » (39531). Je ne pense pas que ce soit un hasard que le premier codicille se termine ici, avec un objet qui soude, pour ceux dont c'est le devoir de se souvenir, celles qui avaient été liées « d'amour » leur vie durant. 


\section{Le sexe des objets}

16 Un autre principe qui règle la distribution des biens se révèle dans ce dernier exemple, principe qui tient non pas à leur ascendance mais, pour ainsi dire, à leur «sexe», ou plutôt au mélange de ces deux ingrédients. Lorsque Louis d'Orléans se voit attribuer la Somme le Roi «qui fut au roy Philippe le Bel»(202), cela permet à Blanche de signaler un croisement entre Valois et Capétiens, dans la mesure où Louis avait obtenu le titre d'Orléans à la mort, en 1392, de Blanche, la fille de Jeanne d'Évreux, au sujet de laquelle la Chronique de Charles VI constate précisément qu'elle était considérée " comme la plus honorable et la plus magnifique des dames du royaume... parce que c'était la seule qui pût se vanter d'être du sang de Philippe le Bel $»^{29}$. Mais c'est en même temps un don à connotation masculine, comme ceux qu'elle avait faits à Charles VI, au frère et aux oncles de celui-ci, tout comme à son neveu Charles de Navarre qui, entre autres, se voit rattaché à son oncle paternel Philippe, frère de Blanche, par le biais d'un « fermail d'or ront ou il y a un gros saphir au milieu », entouré de rubis et de perles et diamants (197).

17 Du côté des femmes prédominent en toute logique les objets à ascendance féminine, provenant de Jeanne de Bourgogne surtout, comme c'est le cas pour un livre d'instruction morale contenant «pleuseurs bons enseignments » (195) que Blanche réserve à Isabeau de Bavière, façon de jeter un pont entre ces deux reines distantes de quatre générations ${ }^{30}$, ou un autre traité didactique à fond orientalisant, le Livre de Josaphat et Barlaam (211), assigné à l'actuelle duchesse de Bourgogne ${ }^{31}$. De Blanche d'Orléans/Jeanne d'Évreux, en revanche, provient un livre «d'oroisons et devocions» $\left(203^{32}\right)$, mais dont la mémoire s'étend plus loin, jusqu'à « la royne Marie » (souvenons-nous que Marie de Brabant aimait fort les beaux manuscrits). Dans ce cas aussi il ne s'agissait pas seulement d'une affaire de générations, car, comme déjà pour la Somme le Roi léguée au mari de Valentine Visconti, ce manuscrit suturait symboliquement la rupture dynastique; cependant, la valeur du livre résidait aussi dans le fait d'avoir été feuilleté jour après jour, année après année, par quatre femmes au moins avant d'échoir à la duchesse d'Orléans ${ }^{33}$. On peut constater un pareil respect du "genre » des objets à l'égard de ses parentes consanguines : ses deux sœurs reçoivent des livres qui avaient appartenu à leur mère - Agnès, un psautier (223), et Jeanne la Jeune, les Heures de Notre-Dame « où nous disons tous les jours noz heures " (230) - tandis que sa nièce, la duchesse de Bretagne, se voit nantie du bréviaire que Blanche avait obtenu à la mort de son autre sœur Jeanne, religieuse à Longchamp (218).

18 Les formules que Blanche adopte pour distribuer ses avoirs les plus chers n'obéissent pas pour autant à une loi rigide, ce qui les rend aptes à suivre des voies de transmission plus imprévisibles. Par exemple, son cousin Louis d'Étampes, obtient trois objets au pedigree féminin, soit un « livre où sont les evangiles des quatre evangelistes, et pluseurs sermons en françois, qui fu à la mère de monseigneur saint Loys de France » (238), ainsi qu'un reliquaire-fermail d'or, décoré d'un cerf dont le corps est fait d'un saphir et qu'entourent trois rubis, trois diamants, treize perles et six émeraudes (237), et un autre reliquaire, composé à nouveau d'un saphir, cette fois en forme de cœur soutenu par deux mains, et contenant des fragments de l'éponge de la Passion et de la tête de saint Jean Baptiste (239), tous deux venant de leur tante commune, Jeanne d'Évreux. Il importe ici de souligner que ce qui fait le "genre » d'un objet est moins ce qu'il est - livre, joyau ou même drap - mais bien plutôt sa provenance. En ceci, les objets ont un avantage sur le trône de France puisqu'ils peuvent s'hériter par lignes féminines aussi bien que 
masculines, et cela de façon équivalente mais non pas indifférente. Au demeurant, la généalogie sexuée constitue la matière explicite du « livre du lignage de Nostre Dame et de ses suers " (214) que Blanche laisse à Marie, duchesse de Bar, comme si elle voulait loger au sein de la famille royale l'idée d'une parenté féminine qui, toute fictive qu'elle soit, n'en est pas moins significative.

\section{Le visible, façon Blanche}

Déplacer des objets sur l'échiquier de la parenté n'est donc pas une opération mécanique, encore moins une procédure qui serait dépourvue d'intentionalité. Blanche, c'est évident, a regardé ses " precieux et nobles jouels » (termes qu'elle emploie au sujet du bréviaire de saint Louis) de très près avant de s'en séparer à jamais. Cette sollicitude à assortir chaque bien avec le destinataire le plus approprié, à marier les objets avec les personnes, se retrouve même dans les legs de choses plus simples, quand, par exemple, elle assigne les seuls draps «à mettre à dames pour leurs gesines [accouchement] » (220) à sa nièce Jeanne, duchesse de Bretagne, qui en l'espace de onze ans avait mis au monde neuf enfants, ou lorsqu'elle choisit la petite Blanche de Navarre comme héritière de sa " chambre » couverte de Bs et de ses armes, chambre que « nous avons nagaires fait faire tout neufve » (410). Ailleurs, ce sont des détails relatifs à l'usage qui retiennent son attention, tel l'anneau avec diamant donné au duc de Bourbon que Philippe VI " portoit en son doy " (206) ou le fermail «que mon dit seigneur et espoux atachoit à sa poitrine» (201) ou encore la chambre verte, donnée à Charles de Rohan, «que l'en tent en nostre hostel quant noz amis viennent devers nous » (240). La teneur strictement artistique peut néanmoins jouer un rôle dans l'évaluation que Blanche fait de ses possessions, l'exemple le plus éclatant étant celui de "nos plus belles heures" héritées de sa mère «à son trespassement » et réservées à celui qui s'y connaissait mieux que nul autre en matière de manuscrits précieux, c'est-à-dire à Jean de Berry ${ }^{34}$ (198). À d'autres reprises, elle utilise le qualificatif «le meilleur», et au sujet de la Somme le Roi elle note que ce «bon livre » est «bien enluminé », l'une des seules instances où les images d'un manuscrit sont prises en compte, raison pour laquelle Richard et Mary Rouse ont récemment proposé de l'identifier avec le superbe manuscrit conservé à la British Library, l'exemplaire princeps enluminé par Maître Honoré à la fin du XIIIe siècle ${ }^{35}$. Et Blanche rapporte même, fait remarquable, un véritable moment d'expertise au sujet du psautier de saint Louis puisqu'elle affirme qu'il avait été authentifié - « c'estoit icellui vraiement » - tant par son mari que par un petit comité, « les femmes de la dicte madame la royne [J. de Bourgogne] qu'il (sic) nous bailla » (200).

20 C'est dire que, si les critères esthétiques entrent en ligne de compte, ils ne sont pas pour autant désintéressés. Ils se mêlent à des logiques venues d'autres horizons, que ce soit le respect de la préséance sociale et des rapports de parenté «objectifs», les considérations de politique familiale ou encore les affinités d'ordre électif. Il y a donc bien là un programme. Mais il est difficile à saisir dans toutes ses nuances, moins en raison de la perte, fréquente, des objets que de l'inadéquation de nos outils conceptuels, le regard que l'histoire de l'art jette sur le passé ayant été presque entièrement accaparé par les seules « images ", peintures, miniatures, sculptures. On comprend la séduction qu'elles exercent parce que, en tant que support d'histoires, elles peuvent être lues comme des véhicules de représentations - d'idées, de goût, de personnalité - alors que les choses sont à nos yeux beaucoup plus muettes, trop engluées dans le matériel. Pour Blanche, il n'y a pas de 
distinction classificatoire entre objet et image, tous deux étant incrustés de sens, d'émotion aussi, à tel point que l'on peut dire que son univers visuel passe autant par le toucher que par le regard, et prend sens là où il acquiert un poids, une épaisseur déployée dans le temps, en amont comme en aval. Observons que, dans la majorité des cas, elle lègue des livres qui ont circulé entre beaucoup de mains, qui ont été recyclés si l'on veut, et aussi des anneaux, joyaux, robes, draps même, qui ont été en contact avec nombre de corps, comme si la valeur d'usage augmentait leur prix plutôt que l'inverse. Du coup, les "objets de mémoire» dont Blanche dispose doivent leur signification moins à leur singularité qu'au fait d'appartenir à un réseau : à l'image de leur propriétaire, ce sont des individus dans un groupe, et à ce titre une approche d'inspiration plus anthropologique semble être mieux à même d'en saisir la portée. D'autant plus que dans cette logique les personnes et les objets se trouvent non pas dans un rapport d'extériorité, mais bien de dépendance mutuelle, à tel point que l'on peut dire que sans objets il ne saurait y avoir de sujets $^{36}$.

Or l'histoire de l'art a toujours privilégié l'étude du singulier - définition même de l'œuvre d'art - et elle a compris le mécénat comme une affaire entre un commanditaire et un artiste auteur d'objets uniques. Si beaucoup d'artistes d'avant-garde du XIVe siècle, tel Jean Pucelle ou son successeur Jean le Noir, ont bien été d'abord les protégés des femmes de la famille royale, il n'en demeure pas moins que, pour Blanche comme pour beaucoup d'autres «mécènes" du Moyen Âge - des femmes surtout -, les relations créatrices passent autant par les morts que par les vivants et produisent des faits sociaux au même titre que des œuvres proprement dites. Ce qui se construit par leurs mains, c'est avant tout une mémoire culturelle inscrite dans la réalité matérielle et dans laquelle privé et public, individu et lignage, féminin et masculin s'enchevêtrent. Ce n'est donc pas par hasard si c'est dans l'acte de tester que Blanche rejoint beaucoup de ses congénères, au statut social d'ailleurs très différent, mais tout aussi sensibles " aux mots et aux choses ». S'érigeant en quelque sorte en centre de triage, elle pouvait répartir des objets qui avaient été les siens temporairement, ce qu'elle ne pouvait faire avec ses terres et autres biens immeubles. Mais cette liberté s'inscrit au sein d'un devoir envers ses ancêtres et ses descendants, si bien que la fama qui en résulterait ne serait pas redevable à une entreprise solitaire (définition moderne du mécénat) mais bien plutôt à la prise en charge d'une memoria collective. Il s'ensuit que transmettre les choses plutôt que d'en être la cause n'était pas pour elle une condition limitative mais au contraire une manière autre, un peu oblique, de s'inscrire dans l'histoire. De même que Blanche utilisa sa position d'intermédiaire comme un instrument de pouvoir, elle aura transformé la distribution des biens en un acte de mécénat. Sous un visage différent, ce mécénat-là n'en fut pas moins une manière de se rendre visible historiquement essentielle pour les femmes.

\section{BIBLIOGRAPHIE}

AUTRAND Françoise, 1994, Charles V le Sage, Paris, Fayard.

--, 1986, Charles VI. La folie du roi, Paris, Fayard. 
BEAUNE Colette, 1985, Naissance de la nation France, Paris, Gallimard.

BEARNE Catherine, 1899, Lives and Times of the Early Valois Queens, Londres, T. Fisher Unwin. BRown Elizabeth A. R., 1991, " Death and the Human Body in the Later Middle Ages : The Legislation of Boniface VIII on the Division of the Corpse ", repr. in The Monarchy of Capetian France and Royal Ceremonial, Aldershot, Variorum.

CAZELLES Raymond, 1958, La Société politique et la crise de la royauté sous Philippe de Valois, Paris, Librairie d'Argences.

--, 1982, Société politique, noblesse et couronne sous Jean le Bon et Charles V, Genève, Droz.

CHIFFOLEAU Jacques, 1980, La Comptabilité de l'au-delà. Les Hommes, la mort et la religion dans la région d'Avignon à la fin du Moyen Âge (vers 1320-vers 1480), Rome, École française de Rome.

CHRISTINE DE PIZAN, 1986, La Cité des Dames, trad. Thérèse Moreau et Éric Hicks, Paris, Stock.

Chronique du Religieux de Saint-Denys, contenant le règne de Charles VI, de 1380 à 1422, 1840, éd. M. L. Bellaguet, t. II, Paris, Impr. de Crapelet.

COSANDEY Fanny, 2000, La Reine de France. Symbole et pouvoir, Paris, Gallimard.

COURTEMANCHE Danielle, 1997, Euvrer pour la postérité : les testaments parisiens des gens du roi au début du XVe siècle, Paris, Harmattan.

DAVIS Natalie Zemon, 2003, Essai sur le don dans la France du XVIe siècle, Paris, Seuil.

DELACHENAL Roland, 1909-1931, Histoire de Charles V, 5 vols., Paris, Picard.

DELISLE Léopold, 1885, « Testament de Blanche de Navarre, Reine de France », Mémoires de l'Histoire de Paris et de l'île-de-France, XII, pp. 1-64.

DOUËT-D'ARCQ L., 1874, Nouveau recueil de comptes de l'Argenterie des rois de France, Société de l'Histoire de France, Paris, Renouard (Inventaire et vente après décès des biens de la reine Clémence de Hongrie, pp. 37-112).

ENGELMANN Jean, 1903, Les Testaments coutumiers au XVe siècle, Paris (repr. Genève, Slatkine 1975). GATOUILlat Françoise, 1999, « La Vierge de Blanche de Navarre et quelques vitraux inédits de la cathédrale d'Évreux ", in Pierre, lumière, couleur. Études d'histoire de l'art du Moyen Âge en l'honneur d'Anne Prache, Paris, Presses de l'Université de Paris-Sorbonne, pp. 309-325.

GUIFFREY Jules, 1894-96, Inventaires de Jean de Berry (1401-1416), Paris, Ernest Leroux.

HEDEMAN Anne D., 1991, The Royal Image : Illustrations of the "Grandes Chroniques de France», 1274-1422 , Berkeley, University of California Press.

HOLLADAY Joan A., 2003, "Fourteenth-Century Queens as Collectors and Readers of Books ", in Books and Readers in the Middle Ages and the Renaissance, Colloque, Université du Tennessee (actes à paraître).

HOWELL Martha C, 1996, «Fixing Movables : Gifts by Testament in Late Medieval Douai», Past and Present, 150, pp. 3-45.

KEANE Rita, 2002, Remembering Louis IX as a Family Saint : Images of Saint Louis created for Jeanne, Blanche, and Marie of Navarre, Thèse de doctorat, Université de Santa Barbara.

KLAPISCH-ZUBER Christiane, 1990a, « Le complexe de Griselda. Dot et dons de mariage », in La Maison et le nom. Stratégies et rituels dans l'Italie de la Renaissance, Paris, Éditions de l'École des Hautes Études en Sciences Sociales, pp. 185-213. 
--, 1990b, «L'invention du passé familial », ibidem, pp. 19-35.

LEBER Constant, 1838, «Le Compte de l'execution du testament et darraine voulenté de feue dame de bonne memoire madame la Royne Jehanne de Evreux ", in Collection des meilleurs dissertations, notices et traités particuliers relatifs à l'histoire de France, t. XIX, pp. 120-169, Paris, Dentu.

LORD Carla, 1997, « Jeanne d'Evreux as a Founder of Chapels : Patronage and Public Piety », in Women and Art in Early Modern Europe : Patrons, Collectors, and Connoisseurs, éd. Cynthia Lawrence, University Park, Pennsylvania State University Press, pp. 21-36.

Mandements et actes divers de Charles V (1364-1380), 1874, éd. Léopold Delisle, Paris, Impr. Nationale.

RAYNAUD Christiane, 1999, "La reine dans les Grandes Chroniques de France », in The Medieval Chronicle, éd. Erik Kooper, Amsterdam, Rodopi, pp. 226-239.

ROUSE Richard et ROUSE, Mary, 2000, Manuscripts and Their Makers : Commercial Book Producers in Medieval Paris (1200-1500), 2 vol., Turnhout, Harvey Miller Press.

schmitт Jean-Claude, 2002, Le corps des images. Essais sur la culture visuelle du Moyen Âge, Paris, Gallimard.

STERLING Charles, 1987, La peinture médiévale à Paris, 1300-1500, t. I, Paris, Bibliothèque des Arts.

De VAIVRE Jean-Bernard, 1981, « Sur trois primitifs français du XIVe siècle et le portrait de Jean le Bon », Gazette des Beaux-Arts, XCVII, n 1347, pp. 131-156.

VAN HOUTS Elisabeth, 1999, Memory and Gender in Medieval Europe, 900-1200, Toronto, University of Toronto Press.

VIGNAT Gaston, 1866, « Notes sur les chapelles absidiales de la basilique de Sainte-Croix d'Orléans », Mémoires de la Société archéologique de l'Orléanais, t. IX, pp. 100-44 (Testament de Blanche d'Orléans, pp. 125-44).

VALLET DE VIRIVILLE, 1858, La Bibliothèque d'Isabeau de Bavière, femme de Charles VI, roi de France, Paris, Techener.

WEINER Annette B., 1976, Women of Value, Men of Renown: New Perspectives in Trobriand Exchange, Austin, University of Texas Press.Trad. fr., 1983, La Richesse des femmes, Paris, Seuil.

DE WINTER Patrick M., 1985, La Bibliothèque de Philippe le Hardi, duc de Bourgogne (1364-1404), Paris, Éditions du Centre National de la Recherche Scientifique.

\section{NOTES}

1. Chronique du Religieux de Saint-Denys 1840 : 659. Le portrait le plus complet de Blanche demeure celui esquissé par Bearne 1899.

2. Telle l'une des verrières, maintenant dépareillée, de la cathédrale d'Évreux montrant Blanche priant devant une très belle Vierge à l'Enfant (analysée par Gatouillat 1999), et quatre statuettes de la Vierge en argent, contenant des reliques, mentionnées tout à la fin du deuxième codicille au testament et que Blanche avait fait exécuter spécialement pour en faire des donations à des églises de son douaire.

3. Respectivement New York, The Cloisters, ms. 54.1.2., et Paris, Bibl. Nat. de France, nouv. acq. lat. 3145. La question de savoir si Blanche avait commandé une traduction du missel et avait été la dédicataire de l'assez obscur Roman de la dame à la licorne n'est pas tranchée (voir de Winter $1985: 129$ et $246-247)$. 
4. Je renvoie aux schémas généalogiques (pp. 61-62) pour plus de précisions sur les individus cités dans ce texte.

5. Le jugement négatif porté sur Charles de Navarre, qui aurait hérité son attitude ambiguë sinon traîtresse face aux Valois de son " âpre » mère, parcourt l'historiographie de cette période. Voir, par exemple, Cazelles 1958 : 205-8, et Autrand $1994:$ 99-108.

6. Dans le testament beaucoup plus succinct de Blanche d'Orléans, sa cousine apparaît en première place parmi ses héritiers et elle reçoit le lot le plus volumineux (Vignat 1866 : 131).

7. Beaune $1985: 140-64$. Sur la présence très réelle des femmes dans les sphères du pouvoir malgré la loi salique, voir Cosandey $2000: 20-8$, qui développe le sentiment de Cazelles 1958 : 284, selon lequel «bien qu'écartées du trône, bien que ne pouvant faire 'le pont et la planche', les femmes de la maison royale sont extrêmement actives et respectées ".

8. Comme par exemple en 1358, lorsque Blanche ouvre les portes de son château de Melun aux troupes navarraises, contribuant ainsi au blocus des voies d'accès à Paris où le dauphin Charles est encerclé. Devenu roi, ce dernier finit par avoir le dessus, contraignant Blanche à lui céder cette ville stratégique et, plus tard, à en soumettre d'autres à la garde des troupes royales.

9. Christine de Pizan souligne le «sage gouvernement» de ces trois princesses dans la Cité des Dames (éd. Moreau et Hicks 1986 : 64-65).

10. Les numéros entre parenthèses renvoient à l'édition de Delisle.

11. De Vaivre 1981, et Sterling $1987: 203-08$ et 166-168, avec discussion de la charte et de la chapelle et résumé des débats sur l'identification des personnages dans quelques peintures supplémentaires, connues par des descriptions et copies plus tardives, contenant des portraits présumés de Blanche, de Philippe VI et de leur fille. Je remercie Rita Keane d'avoir mis à ma disposition le chapitre de sa thèse traitant de Blanche de Navarre. Nos informations proviennent en premier lieu de la description de Saint-Denis publiée en 1635 par Jacques Doublet. Pour la chapelle de Jeanne d'Évreux, voir Lord 1997, et aussi Beaune 1985 : 120-123.

12. Mandements 1874 : no. 225A.

13. Paris, Bibl. Nat. de France, ms. fr. 2813, fol. 395. Delachanal 1909, I : 40-42 ; ainsi que Hedeman 1991 : 95-133, et Raynaud 1999. Les reines interviennent à plusieurs reprises dans les années suivantes, et occupent les places d'honneur dans la grande assemblée réunie au Palais de Paris en 1380 où se décidèrent les modalités de la régence à la suite de la mort de Charles $\mathrm{V}$.

14. Engelmann 1903 ; Courtemanche 1997 ; et, pour une vue plus élargie, Chiffoleau 1980.

15. Chronique du Religieux de Saint-Denys 1840 : 657-63. Dans le même passage, l'auteur se plaint du fait que celle qui s'était enrichie sans opprimer ses sujets, qui était si prodigue dans ses œuvres charitables, n'avait pas suffisamment pourvu Saint-Denis. Il nous informe aussi qu'en réalité lui furent accordés les honneurs royaux lors de ses funérailles en dépit du fait que Blanche n'avait été ni couronnée ni sacrée.

16. Comme l'a montré Brown 1991, Blanche de Navarre se conforme, avec quelques autres rares membres de la famille royale, à l'interdit de Boniface VIII, émis en 1299 dans la bulle Detestande feritatis.

17. À mesure que l'on descend la hiérarchie sociale apparaissent les dons en numéraire et des livres moins ornés, des joyaux moins coûteux, des robes tant longues que courtes, un peu de vaisselle et de meubles, sans oublier les chevaux et muids de blé et queues (futailles) de vins dont Blanche gratifie certains de ses serviteurs, ou encore le restant des fils, aiguilles, pièces d'étoffes que ses ouvrières en soie sont autorisées à emporter.

18. Holladay 2003 : 11. Bien sûr, ces chiffres ne tiennent jamais compte des possessions données ou perdues avant la rédaction du testament ou de l'inventaire après décès. Ainsi, lors du siège de Mantes, en 1364, la demeure de Blanche fut pillée par les Bretons, et plusieurs importants joyaux lui furent dérobés, perte pour laquelle Charles V lui paya 1000 francs de réparation.

19. Howell $1996: 38$. 
20. Probablement Marguerite (m. 1417), personnage en vue à la cour de Charles VI, veuve de Jacques Ier, sieur de Préaux, cousin germain de Louis II de Bourbon, et comme celui-ci à la fois intime de Charles V mais aussi proche du «parti navarrais».

21. Delisle ne s'y était pas trompé lorsqu'il écrivait qu'il « est impossible de n'être pas frappé du soin qu'elle prend de faire l'histoire de ses volumes les plus précieux » (Delisle $1885: 2$ ).

22. Ces articles semblent correspondre à ceux décrits, avec quelque variation, dans l'exécution testamentaire de Jeanne d'Évreux (Leber 1838: 167). En revanche, le testament de Blanche ne mentionne pas « l'aigle d'or qui tient en son bec une jointe de monseigneur saint Denys qui fut a la royne de Navarre sa mere ", que Jeanne d'Évreux lui laisse pareillement.

23. Cela n'est pas sans rappeler le caractère provisoire des cadeaux des maris florentins à leurs nouvelles épouses analysé par Klapisch-Zuber 1990a.

24. Le fermail est une broche ou agrafe. Philippe avait acheté douze fermails dans la succession de Clémence de Hongrie, mais aucun ne mentionne des reliques (Douët-d'Arcq 1874 : 42-43).

25. La mémoire que Blanche a de cet objet s'arrête à son aïeul illustre, même s'il s'agit d'un manuscrit plus ancien, datant de la fin du XIIe siècle, anglais de surcroît. Il a été de longue date identifié avec le psautier conservé à Leyde, Bibliothèque de l'Université, ms. Latin 76A. Voir de Winter 1985 : 260-61. Jeanne d'Évreux possédait également un "psautier de saint Louis », qui entra dans la collection de Charles V et se trouve aujourd'hui à la Bibl. Nat. de France, ms. lat. 10525.

26. Elle consulte les Grandes Chroniques. Le rôle d'honneur dans cette cérémonie lui fut d'ailleurs accordé ainsi qu'à Blanche d'Orléans à qui on avait acheté la robe - toute imprégnée de légitimité capétienne - portée par Isabeau.

27. Toute collection qui se respectait devait avoir quelques souvenirs et, mieux encore, des reliques de saint Louis. Blanche légua aussi un reliquaire d'argent avec une «jointe » de saint Louis et un livre de sa vie en français «aux dames» de l'Hôtel-Dieu de Vernon «pour avoir mémoire de Saint Louis » (35).

28. Klapisch-Zuber 1990b : 26-28; et pour les objets comme des chevilles de la transmission des savoirs généalogiques faite par les femmes, van Houts 1999 : 93-120.

29. Chronique du Religieux de Saint-Denys, p. 63. La succession Orléans fut l'occasion pour Charles V d'étendre aux apanages les règles en vigueur dans le domaine royal, à savoir que les filles ne peuvent ni les hériter ni les passer à leurs descendants.

30. Ce manuscrit n'apparaît pas parmi ceux mentionnés dans l'étude de Vallet de Viriville 1858.

31. De Winter $1985: 137$.

32. Sans doute le même que le testament de Blanche d'Orléans décrit comme « un petit livret d'oroisons qui fu à la Royne Jehanne sa mère » (Vignat $1866: 131$ ).

33. Pour être exact il faut ajouter que Blanche changea d'avis deux ans plus tard en lui substituant un autre livre, «nostre breviaire à l'usage de Romme, qui est en deux volumes, qui fu ma ditte dame la royne Jeanne » (405).

34. Ce manuscrit reste non identifié, et Blanche n'apparaît guère dans les inventaires du duc publiés par Guiffrey 1894-96.

35. Rouse et Rouse 2000, t. I, pp. 148-54. Il s'agit du manuscrit Add. 54180, discuté aussi dans Sterling 1987 : 44-47.

36. Je pense surtout aux travaux de Weiner 1976, qui a bien mis en évidence le rôle des dons faits par les femmes lors des moments de rupture dans le cycle de vie et comment ceux-ci, en tant que principe régénérateur de l'identité matrilinéaire, participent à la structuration socio-économique de la société dans son ensemble. Pour les objets, artistiques et autres, dans les pratiques du don, voir Davis 2003 et pour une conception élargie de l'imago et des objets dans l'univers médiéval, Schmitt 2002. 


\section{RÉSUMÉS}

Contrairement à ce que l'on entend généralement par mécénat - la commande d'images, peintes ou sculptées, passée entre deux individus vivants - on propose ici d'en élargir les termes pour y englober une autre modalité de la circulation des biens culturels : l'héritage, particulièrement significatif pour les femmes, qui disposaient souvent d'un important patrimoine artistique sans en avoir été les commanditaires. En examinant la manière dont Blanche de Navarre ( $† 1398)$, épouse de Philippe VI de Valois et reine douairière pendant près d'un demi-siècle, disposa de ses objets les plus précieux, joyaux et livres, dans un testament exceptionnel par son détail, l'accent est mis sur l'acte de tester comme un moment créateur, sinon d'objets, du moins de faits sociaux. Car, par la distribution savante de ses objets, Blanche construisit, pour ses successeurs, une mémoire, solidifiée dans des choses, des liens unissant les membres épars, vivants ou morts, de sa parenté.

Modern notions of patronage focus on the commission of images, painted or sculpted, contracts that bind two living individuals. We propose to enlarge these terms to include another modality by which cultural goods circulate: inheritance, especially significant for women, who often found themselves in possession of an important artistic patrimony that they had not commissioned. Examination of the exceptionally detailed testament of Blanche de Navarre (d. 1398), wife of Philip VI of Valois and dowager queen for almost half a century, shows that the bequest of her most precious objects, jewels and books alike, was an occasion to create meaningful social facts. For it is through the attentive distribution of her objects that Blanche was able to memorialize for her successors, in concrete things, the ties that bound her scattered kin, living and dead.

\section{INDEX}

Mots-clés : joyaux, manuscrits, mécenat, objets d'art, parenté, testament, transmission, Reine de France

\section{AUTEUR}

\section{BRIGITTE BUETTNER}

Brigitte BUETTNER enseigne l'histoire de l'art médiéval à Smith College (Massachusetts, USA). Spécialiste des manuscrits profanes de la fin du Moyen Âge, ses publications comprennent notamment Boccaccio's «Des cleres et nobles femmes »: Systems of Signification in an Illuminated Manuscript (Seattle, Washington University Press, 1996) et « Past Presents : New Year's Gifts at the Valois Courts, ca. 1400 », Art Bulletin, 83, 2001, pp. 598-625. Elle prépare actuellement une étude sur les pierres précieuses et l'imagination minéralogique au Moyen Âge. 